\title{
Magnetic topology of coronal mass ejection events out of the ecliptic: Ulysses/HI-SCALE energetic particle observations
}

\author{
O. E. Malandraki ${ }^{1,2}$, E. T. Sarris ${ }^{1}$, and G. Tsiropoula ${ }^{2}$ \\ ${ }^{1}$ Democritus University of Thrace, Department of Telecommunications and Space Physics, Xanthi, 67100, Greece \\ ${ }^{2}$ National Observatory of Athens, ISARS, Athens, 15236, Greece
}

Received: 21 August 2002 - Revised: 15 April 2003 - Accepted: 13 May 2003

\begin{abstract}
Solar energetic particle fluxes $\left(E_{e} \geq 38 \mathrm{keV}\right)$ observed by the ULYSSES/HI-SCALE experiment are utilized as diagnostic tracers of the large-scale structure and topology of the Interplanetary Magnetic Field (IMF) embedded within two well-identified Interplanetary Coronal Mass Ejections (ICMEs) detected at $56^{\circ}$ and $62^{\circ}$ south heliolatitudes by ULYSSES during the solar maximum southern high-latitude pass. On the basis of the energetic solar particle observations it is concluded that: (A) the high-latitude ICME magnetic structure observed in May 2000 causes a depression in the solar energetic electron intensities which can be accounted for by either a detached or an attached magnetic field topology for the ICME; (B) during the traversal of the out-of-ecliptic ICME event observed in July 2000 energetic electrons injected at the Sun are channeled by the ICME and propagate freely along the ICME magnetic field lines to $62^{\circ} \mathrm{S}$ heliolatitude.
\end{abstract}

Key words. Interplanetary physics (energetic particles; interplanetary magnetic fields)

\section{Introduction}

The topology of magnetic field lines carried into the interplanetary medium by coronal eruptive events has been investigated for several decades. Cocconi et al. (1958) and Gold (1959) suggested the presence of huge magnetic loops that could retain their connection to the Sun and extend to distances beyond the Earth's orbit. Furthermore, it has been conjectured that magnetic loops could even be detached from the Sun to form "magnetic bubbles" (Piddington, 1958). Gosling et al. (1974), using Skylab measurements, observed Coronal Mass Ejections (CMEs) that appeared as large magnetic loops anchored near regions with strong magnetic fields at the solar surface, yet expanding outward through the solar corona.

Correspondence to: E. T. Sarris (sarris@ee.duth.gr)
Between $1 / 3$ and $1 / 2$ of all CMEs in the solar wind are also magnetic clouds (Gosling, 2000), characterized by a flux rope geometry observed as a large, smooth rotation in the magnetic field, a strong magnetic field and a low proton temperature (Klein and Burlaga, 1982; Osherovich and Burlaga, 1997).

The first attempt to probe the topology of interplanetary fields by means of solar energetic particles (SEPs) was made by Rao et al. (1967). They found four periods of bidirectional anisotropies of $10-\mathrm{MeV}$ protons as part of a survey of energetic storm particle events observed by Pioneer 6 and 7. They argued that a Gold bottle (i.e. attached magnetic bottle geometry) could not account for their observations which were better explained by particles behind a blast wave in an open field line configuration. Palmer et al. (1978), who made the next attempt to deduce magnetic field topologies from energetic particle measurements, found 16 periods of bi-directional fluxes of low-energy solar particles with an average duration of $9 \mathrm{~h}$. They argued in favor of a Gold bottle in contrast to Rao et al. (1967). Kutchko et al. (1982) analyzed a $1-\mathrm{MeV}$ proton and alpha SEP event on 12 October 1977, that showed field-aligned bi-directional fluxes for about $5 \mathrm{~h}$. Electron anisotropies were also bi-directional but were peaked perpendicular to the magnetic field. As candidate topologies for the IMF they compared the Gold bottle with a plasmoid, and the former configuration was considered to be the most consistent. Sarris and Krimigis (1982) have presented IMP-7 observations of energetic particles injected by solar flares into extended solar magnetic loop-like structures. From the time history of the angular distributions of the particle intensities they have inferred that energetic particles are bouncing between two mirrors located at the Sun and have obtained for the first time estimates of the extent of magnetic loops to distances $\sim 3.5 \mathrm{AU}$ from the Sun. The results of a comprehensive survey of low-energy ion bi-directional anisotropies and their relation to isolated interplanetary magnetic structures, as observed by ISEE-3 at the time of peak activity of sunspot cycle 21 , have been presented by Marsden et al. (1987). From a comparison of 


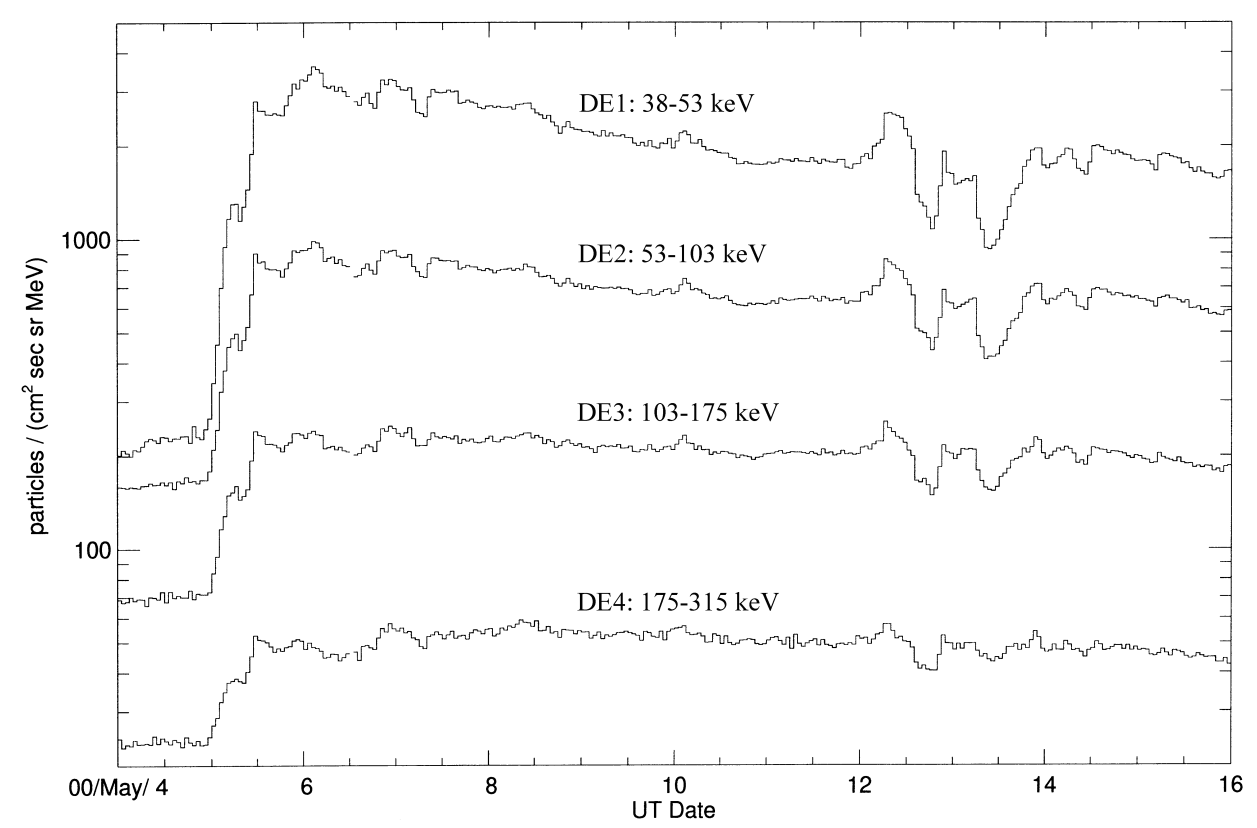

Fig. 1. Overview of $38-315 \mathrm{keV}$ hourly-and spin-averaged intensities of magnetically deflected electrons as measured by the WART B detector of the HI-SCALE experiment for the interval 4-16 May 2000, during a major solar flare electron event.

the anisotropy signatures at 35 and $620 \mathrm{keV}$ they concluded that their observations were most consistent with the transient magnetic structures being detached bubbles consisting of closed loops or tightly wound cylindrical helices, rather than extended tongue-like loops attached to the Sun at the time of observation.

Occasionally, the onset of a solar particle event is observed by a spacecraft located inside an ICME (Kahler and Reames, 1991; Farrugia et al., 1993; Mazur et al., 1998; Malandraki et al., 2000a, 2002). Such observations suggest that at least one end of the field lines embedded within the ICME is connected to the Sun, rather than detached. The onsets discussed in these studies were observed in the ecliptic within 1.5 AU from the Sun. Armstrong et al. (1994) observed hot $(\sim 270 \mathrm{keV})$ coronal particles at $4.6 \mathrm{AU}$ and $32^{\circ}$ south heliolatitude, during the passage of a CME which had an internal structure of a large magnetic flux rope. They concluded that the beams observed by the ULYSSES/HI-SCALE instrument can be explained if the flux rope were rooted near a coronal energetic particle source, thus providing a "conduit" for these particles along its axis. Moreover, Pick et al. (1995) have identified at $3.5 \mathrm{AU}$ and $54^{\circ}$ south heliolatitude a particle propagation channel inside an ICME which was filled by solar flare particles. These out-of-ecliptic observations by ULYSSES near solar minimum suggest that ICME field lines at high latitudes and large distances from the Sun may continue to be rooted at the Sun.

Bothmer et al. (1996) observed bi-directional and streaming anisotropy suprathermal electron and $0.4-5 \mathrm{MeV}$ ion flux characteristics within two different portions of an ICME detected by ULYSSES, which they interpreted as evidence of closed magnetic loops and open magnetic field lines, respec- tively. Furthermore, Malandraki et al. (2000b) have examined the time evolution of the angular distributions of the solar electron fluxes ( $E \geq 42 \mathrm{keV}$ ) observed during the passage of two ICMEs over ULYSSES in March 1991. The investigation revealed that both ICMEs presented a filamentary structure comprising magnetic filaments with distinctly different electron anisotropy characteristics. The ICMEs were found to be still anchored to the Sun when they arrived at ULYSSES and involved both open and closed magnetic field topologies, probably resulting from sustained 3-dimensional magnetic reconnection at the Sun (Gosling et al., 1995). Recently, Malandraki et al. (2001) have presented observations of unidirectional $38-315 \mathrm{keV}$ electron anisotropies outside and within an ICME that was convected over ULYSSES in April 1998 when the spacecraft was at a 5.4 AU helioradius. They suggested that the most plausible interpretation of the observations is that a large portion of the ICME and its environs consisted of open IMF filaments rooted at the Sun at only one end threading through the ICME. On the other hand, the unusual electron bi-directional anisotropies observed in the leading portion of the ICME provided evidence for trapped electrons along closed loop-like structures anchored at both ends to the Sun.

Larson et al. (1997) have presented WIND observations of five impulsive $\sim 1-10^{2} \mathrm{keV}$ solar flare electron events inside the magnetic cloud observed upstream of the Earth on 18-20 October 1995. Although the cloud magnetic field exhibited the smooth, continuous rotation signature of a helical flux rope, the $\sim 0.1-1 \mathrm{keV}$ heat flux electrons and $\sim 1-10^{2} \mathrm{keV}$ energetic electrons showed numerous simultaneous abrupt changes from bi-directional streaming to unidirectional streaming to complete flux dropouts. They have 


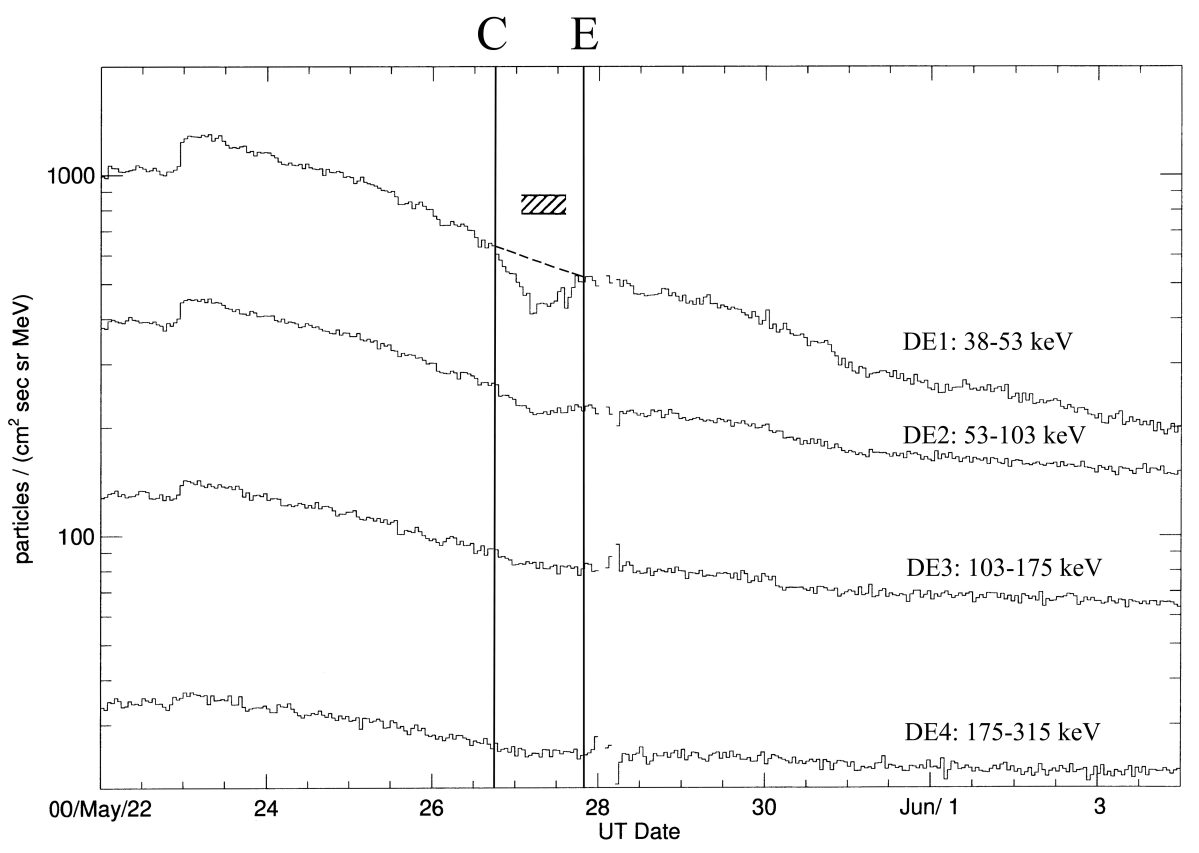

Fig. 2. Vertical traces $\mathrm{C}$ and $\mathrm{E}$ denote the commencement and end of an ICME observed by ULYSSES at $56^{\circ}$ south heliolatitude during the solar maximum southern high-latitude pass.

interpreted these observations as evidence that the cloud comprised magnetic field lines connected to the Sun on both ends, on one end, and completely disconnected, intertwined together. Out of the ecliptic plane, Malandraki et al. (2001) have examined the intensities and directional distributions of a solar electron event observed during the traversal of an ICME over ULYSSES in January 2000 at $43^{\circ}$ south heliolatitude and $\sim 4.1$ AU helioradius. Based upon the observation of energetic electrons streaming for long intervals $(\sim 34 \mathrm{~h}$ in total) in a collimated beam along IMF lines they concluded that open IMF filaments connected to the Sun at only one end are threading through a large portion of the ICME. However, the observation of a 3-h long abrupt depression in the event flux profile with bi-directional electron anisotropy signatures throughout it indicated that injected particles at the Sun, could not enter this ICME portion which was thus concluded to be disconnected from the Sun exhibiting a plasmoid topology. Notably, this event, observed near solar maximum during the second ULYSSES southerly high-latitude pass, constitutes the first observation of an out-of-ecliptic ICME event comprising complex intertwined structures that include regions both connected to and disconnected from the Sun.

In this work, we use solar energetic electron fluxes $\left(E_{e} \geq 38 \mathrm{keV}\right.$ ) observed by the ULYSSES/HI-SCALE instrument, in order to probe the internal magnetic structure of two well-identified ICME events detected at $56^{\circ}$ (May 2000) and $62^{\circ}$ (July 2000) south heliographic latitudes during the ULYSSES second ascent to southern high-latitude regions of the heliosphere. The still unsolved problem of whether ICMEs have been detached from the solar corona or are still magnetically anchored to it when they arrive at the spacecraft is rigorously tackled.

\section{Instrumentation}

In this work, hourly-averaged measurements of the angular distributions of the intensities of energetic electrons in the energy range $42-178 \mathrm{keV}$, detected by the sunward looking telescope LEFS60 (Low-Energy Foil Spectrometer) of the HI-SCALE experiment (Heliosphere Instrument for Spectra, Composition and Anisotropy at Low Energies) on board ULYSSES, are utilized. The LEFS60 telescope has a geometrical factor of $\sim 0.48 \mathrm{~cm}^{2}$ sr. The number 60 denotes the angle that the collimator centerline of the telescope makes with the ULYSSES spin axis. The HI-SCALE experiment has been described in full detail by Lanzerotti et al. (1992). In the sunward-looking LEMS30 ion telescope (Low-Energy Magnetic Spectrometer), mounted at $30^{\circ}$ with respect to the spacecraft axis, electrons with energies below $\sim 300 \mathrm{keV}$ are swept away from the ion detectors of the telescope and counted by the separate detector B, of the composition aperture (CA; sometimes referred to in the ULYSSES project as the "WART"). Hourly and spin-averaged measurements of these magnetically deflected electrons (DE) in the energy range DE1-DE4 $(38-315 \mathrm{keV})$ are also reported. The WART B detector head has a geometrical factor of $\sim 0.05 \mathrm{~cm}^{2} \mathrm{sr}$.

The ULYSSES magnetic field experiment consists of a fluxgate magnetometer (FGM) and a vector helium magnetometer (VHM) which measure the three orthogonal components of a magnetic field vector (Balogh et al., 1992). 


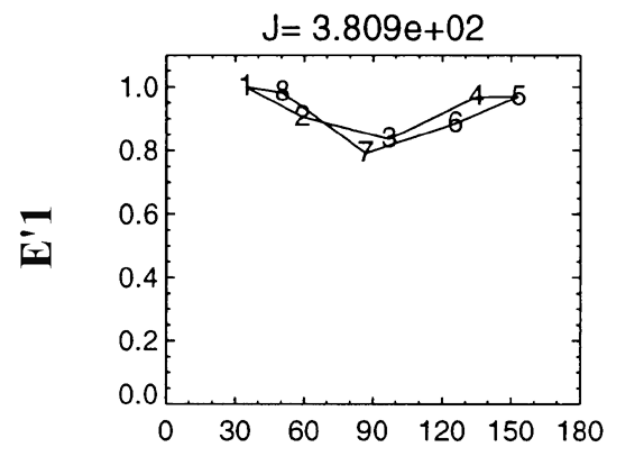

00 May 27 05:00

00 May 27 06:00

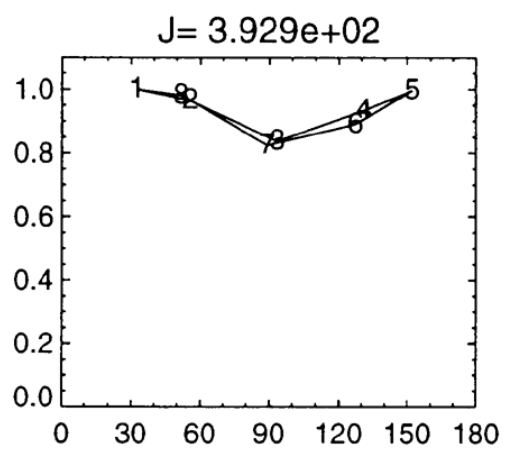

00 May 27 06:00

00 May 27 07:00

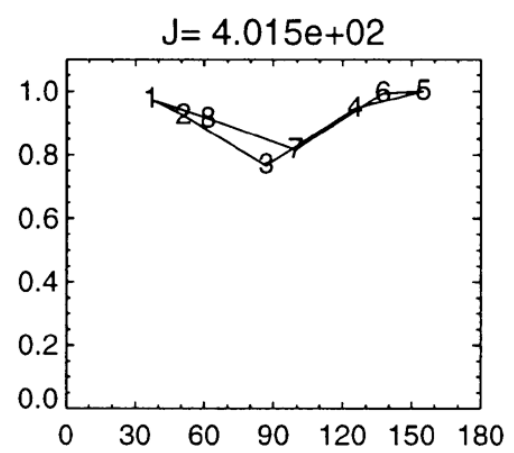

00 May 27 09:00

00 May 27 10:00

Fig. 3. Hourly-averaged electron pitch-angle distribution snapshots observed by ULYSSES inside the May 2000 high-latitude ICME (E'1: $42-65 \mathrm{keV})$.

Hourly-averaged IMF measurements are used. Solar wind data were provided by the SWOOPS experiment (Solar Wind Observations Over the Poles of the Sun) on board ULYSSES (Bame et al., 1992). Information on solar activity is provided by the Solar Geophysical Data monthly report (No. 677, Part II, July 2000).

\section{Observations and data analysis}

Hourly-and spin-averaged differential intensities of electrons in four energy channels $(38-315 \mathrm{keV})$ as measured by the WART B detector head of the HI-SCALE experiment from 4-16 May 2000 are presented in Fig. 1. A major solar electron event is observed. On 4 May a M6.8 X-ray flare starting at 10:57 UT and located at S17 W90 was observed. The origin of the electrons could be either a flare-related acceleration mechanism (Simnett, 2001) or some other accelerating mechanism near the Sun (e.g. acceleration by a CMEdriven shock near the Sun). The intensity variations on 1214 May are observed during the passage of an ICME event (not shown) over ULYSSES (Gosling and Forsyth, 2001). In Fig. 2, electron fluxes are shown later, during the decay phase of the electron event. An ICME, bounded by vertical traces C (Commencement) and E (End), began at 18:00 UT on 26 May and ended at 20:00 UT on 27 May (see Table 1 of Gosling and Forsyth, 2001). The ICME did not produce a shock wave disturbance in interplanetary space. At this time, ULYSSES was climbing towards the south polar latitudes and was located at $56^{\circ}$ south heliolatitude and at a $\sim 3.45$ AU helioradius. As it can be seen in Fig. 2, the electron intensities are observed to be smoothly decaying until ULYSSES crosses the front boundary of the ICME. Upon entry within the ICME, the smooth decay phase of the electron event is interrupted and an abrupt flux depression is observed throughout the ICME passage. This effect is mostly evident in the lower energy (DE1: 38-53 keV) electron intensities. At the trailing edge of the ICME the electron intensities at- tain the values they would have had if the decay phase of the event had proceeded smoothly without interruption (dashed line).

Careful inspection of the pitch-angle distributions (PADs) of the electron intensities observed during the passage of the ICME over ULYSSES reveals the existence of unusual particle anisotropies. Figure 3 shows snapshots of the hourly-averaged electron angular distributions as observed by ULYSSES inside the ICME. Normalized differential flux is plotted versus the pitch angle. $\mathrm{J}$ on top indicates the maximal flux to which the distribution is normalized. The time interval is indicated below each graph. As depicted in Fig. 3, distinct electron bi-directional PADs, that persist for $13 \mathrm{~h}$ (hatched horizontal bar in Fig. 2), are observed in the heart of this high-latitude ICME.

The energetic electron observations during the traversal of another out-of-ecliptic ICME event, detected by Ulysses on its way towards the Sun's south pole in July 2000, are now presented and analyzed. In Fig. 4, an overview of the hourly-and spin-averaged differential intensities of energetic electrons $(38-315 \mathrm{keV})$ is shown for the interval 4 to 28 July 2000. An ICME extending from 00:00 UT on 15 July (C) until 02:00 UT on 16 July (E) is observed during this period (Gosling and Forsyth, 2001), 6 days after the passage over Ulysses of a Forward (FS) - Reverse (RS) shock pair bounding a Corotating Interaction Region (shock list at http://swoops.lanl.gov). The ICME had no associated shock and was observed during a major solar electron event.

In Fig. 5, we focus on the ICME and its vicinity. On the top panel, 30-min averaged electron intensities are shown for the interval 11-18 July 2000. The three lower panels present hourly-averaged magnetic field magnitude and direction in the heliocentric RTN system for the same period. The RTN coordinate system uses the Sun-ULYSSES direction $(R)$, prograde heliocentric orbital direction $(T)$, and the right-hand completion $(N)$. Evidently, the ICME consisted of an isolated magnetic field structure. A noteworthy feature is the very stable nature of the magnetic field inside the 


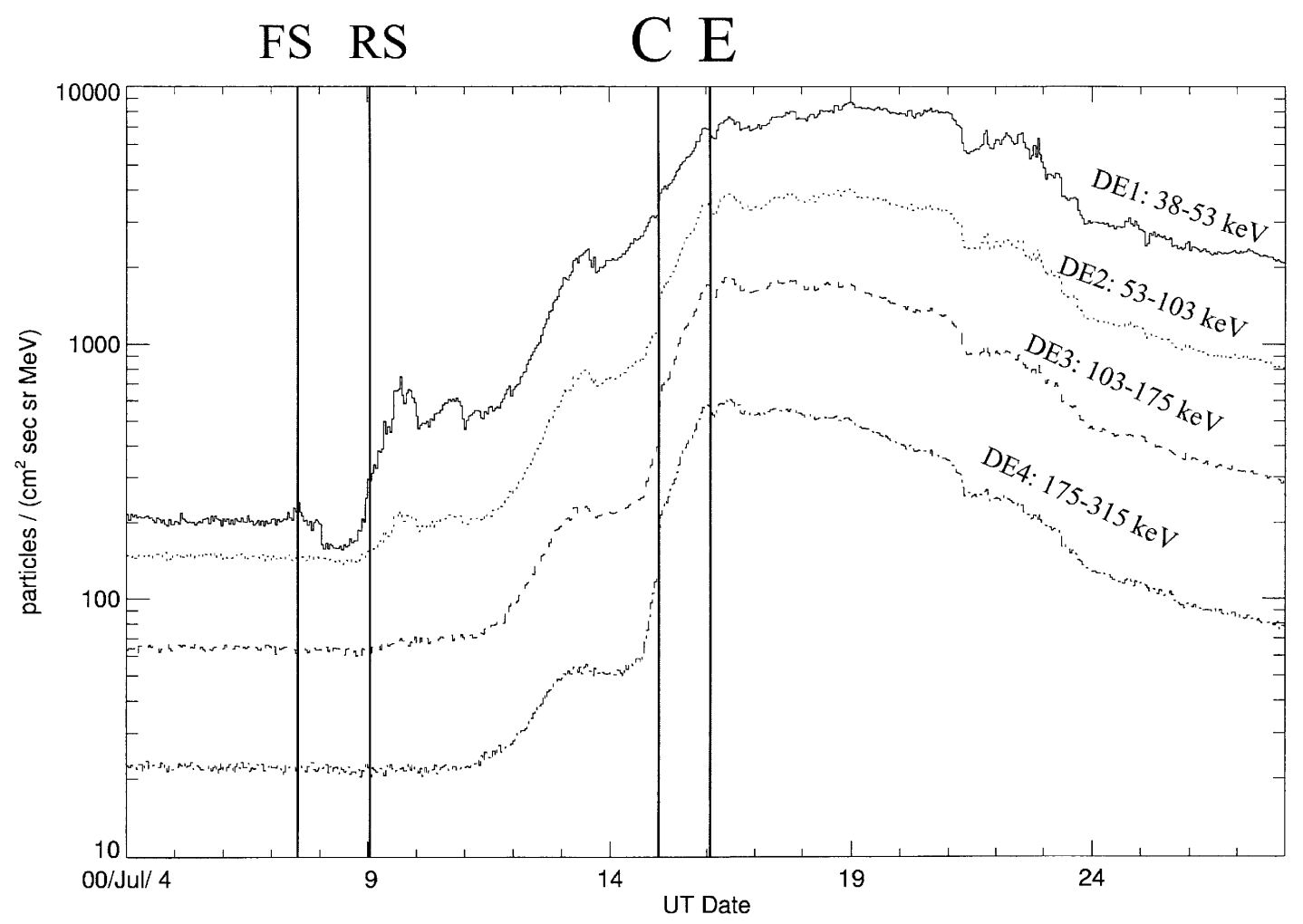

Fig. 4. An ICME was observed by ULYSSES at $62^{\circ}$ south heliolatitude during the major solar electron event associated with the Bastille Day solar flare.

ICME compared to the turbulent field observed outside. The magnetic field magnitude remains nearly the same, and the azimuth angle is constant throughout the ICME, whereas the observation of a smooth rotation in the field polar angle is a signature that the ICME has a flux rope structure (Gosling and Forsyth, 2001).

The electron fluxes in the higher energy channels start an additional rise at 16:00 UT on 14 July. During this period ULYSSES was located at $62^{\circ}$ south heliolatitude, at $3.2 \mathrm{AU}$ helioradius and $116^{\circ}$ east from the Earth. Using the measured solar wind velocity of $580 \mathrm{~km} / \mathrm{s}$ by the SWOOPS experiment on board ULYSSES at the onset of the event, the Parker spiral connecting ULYSSES to the Sun was computed to be $\sim 3.7 \mathrm{AU}$ long and mapped back to $\sim \mathrm{S} 62 \mathrm{~W} 19$ on the visible hemisphere of the Sun as viewed from the Earth. The transit time for a $175-315 \mathrm{keV}$ electron with a $60^{\circ}$ pitch angle (FWHM of the PADs at 01:00 UT on 15 July) along the $\sim 3.7$ AU long spiral is $1.5 \mathrm{~h}$. Thus, an injection time at the Sun at 14:30 UT on 14 July would be implied. On 14 July active region 9077 on the Sun produced the large Bastille Day solar flare of X5.7 intensity (white light Ha importance 3B), which started at 10:03 UT and was located at N22 W07 and also a much less intense 1N/M3.7 solar flare at N20 W08 starting at 13:44 UT (Fig. 5, arrows on top panel). Obviously, there was a difference of $\sim 84^{\circ}$ in heliolatitude between the magnetic connection of ULYSSES to the Sun and the flare locations. The delayed onset and slow rise time of the electron event are to be expected from the consistently "poor" nominal connection with the flare latitudes. A flare-related electron acceleration mechanism implies that the electrons have undergone drifts across the highly inhomogeneous fields near the Sun before reaching the ULYSSES footpoint and escaping in space, populating this high-latitude ICME. However, some other acceleration mechanism near the Sun (e.g. acceleration by a CME-driven shock) which feeds the magnetic field lines threading through the ICME with solar energetic electrons is another probably more likely scenario to account for the origin of the electrons and their spatial distribution.

Representative snapshots of the angular distributions of the energetic electron intensities detected by ULYSSES throughout the ICME and its vicinity are presented in Fig. 6. The first three columns in Fig. 6 show snapshots of the isotropic PADs observed by ULYSSES after the onset of the electron event outside the ICME. ULYSSES detects a transition after entering the ICME: isotropic PADs outside suddenly switch to unidirectional inside, indicating that electrons streaming away from the Sun are observed inside the ICME. Furthermore, upon entry into the ICME and coincident with the dramatic change in the electron PADs, an abrupt and discontinuous increase in the particle intensities is observed (Fig. 5). We believe this indicates that the magnetic connection of ULYSSES to the acceleration source is greatly improved inside the ICME. Unidirectional PADs, indicating electrons streaming away from the Sun are detected, 

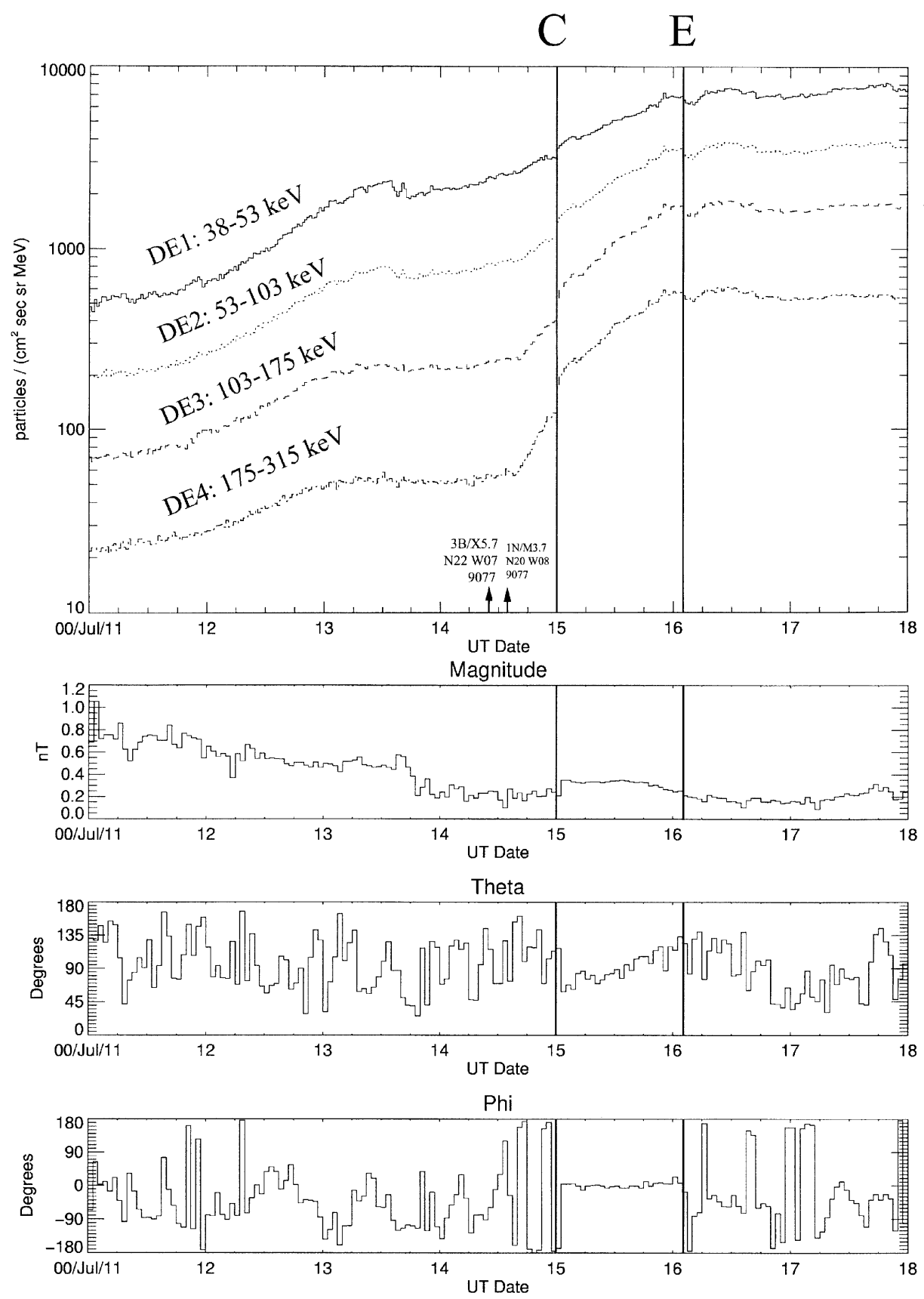

Fig. 5. ULYSSES energetic electron and magnetic field data in the vicinity and during the traversal of the ICME observed in July 2000. An abrupt and discontinuous increase in the particle intensities is observed upon entry of ULYSSES inside the ICME.

are persistently observed for $4 \mathrm{~h}$ after the entry of ULYSSES within the ICME and from 16:00 UT on 15 July, until exit from the ICME. In between these time intervals, PADs also exhibit a clear tendency to unidirectionality. The PADs become isotropic upon exit of the spacecraft from the ICME.

\section{Summary and conclusions}

Large-scale magnetic structures of the interplanetary space cannot be uniquely determined from local plasma and field measurements alone (e.g. Gosling and Roelof, 1974). In this respect, solar energetic protons and relativistic electrons provide an excellent means for remote sensing diagnostics of such structures and are thus valuable probes of ICME internal magnetic structure and topology (Sarris and Krimigis, 1982; Richardson, 1997). Their gyroradii are small compared to ICME scale sizes, while their speeds are very much greater than ICME speeds. In addition, they propagate nearly "scatter-free" in the unshocked IMF, i.e. with negligible cross field diffusion and large mean free paths along the 


\section{OUTSIDE ICME}
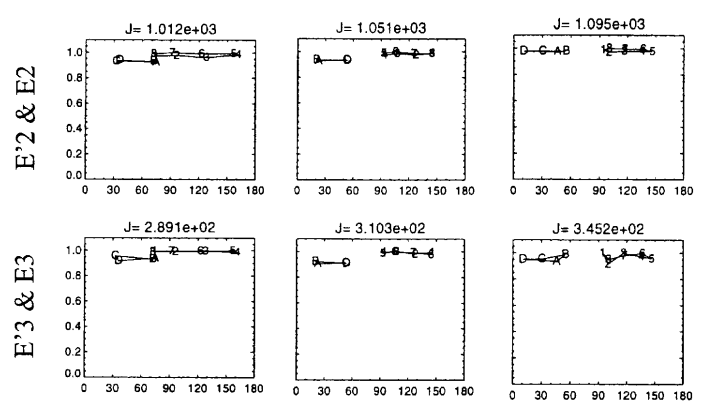

00 Jul $1420: 00$ 00 Jul 14 21:00

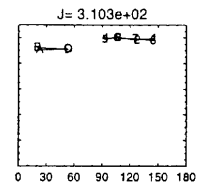

00 Jul 14 21:00 $00 \mathrm{Jul} 14$ 22:00

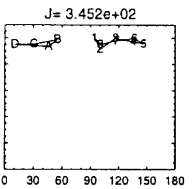

00 Jul 14 23:00 00 Jul 15 00:00
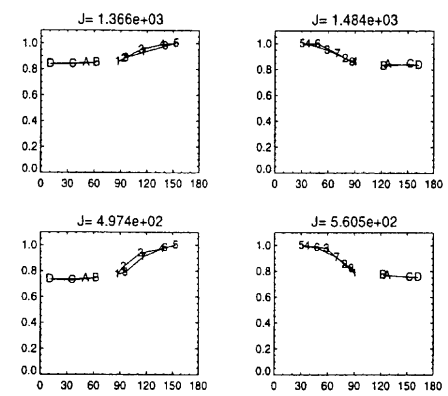

00 Jul 15 00:00 00 Jul $1501: 00$

\section{NS I D E I C ME}

Fig. 6. Representative snapshots of the electron pitch-angle distributions observed inside and outside the high-latitude ICME by the ULYSSES/HI-SCALE instrument in July 2000 (E'2: 65-112 keV, E'3: 112-178 keV).

field. These propagation characteristics provide the means for tracing the large-scale structure of ICMEs by unraveling observations of the angular distributions of the intensities of the energetic particles during the ICME passage (Sarris and Krimigis, 1982).

An abrupt depression in the low-energy electron intensities that interrupted the solar electron event decay phase has been observed by ULYSSES during the passage of the highlatitude ICME detected at $56^{\circ}$ south heliolatitude in May 2000. As the leading edge of the ICME is convected over ULYSSES the decaying electron fluxes at the highest energies have already nearly reached pre-event ambient flux values (see Fig. 1). Under such conditions, a flux depletion in the electrons at these energies would probably be impossible to observe. Based upon the low-energy electron observations during this period we conclude that electrons injected at the Sun cannot enter within this out-of-ecliptic ICME, suggesting probable disconnection of the ICME from the Sun. The electrons observed within this ICME may have been initially injected at the Sun before disconnection occurred. Gosling et al. (1995) suggest that 3-D magnetic reconnection can produce detached U-shaped magnetic structures connected to the outer heliosphere at both ends. On the basis of the observation of bi-directional electron intensities within the large middle portion of the ICME for $13 \mathrm{~h}$, a plasmoid-type magnetic structure which has severed magnetic connection with the Sun cannot be definitely ruled out for this ICME portion. If SEPs are injected into interplanetary space while a plasmoid is present in space, those particles should be excluded from the region of the plasmoid, as they would have to propagate across the magnetic field in order to enter it. Thus, the subsequent passage of such regions past the spacecraft should be accompanied by a substantial reduction in SEP fluxes, in agreement with what is observed by ULYSSES for this event.

However, as it is not known from the observations if the electron flux depletion would extend to higher energies or not (Lin and Kahler, 1992), it is also highly probable that the counterstreaming electrons observed in the ICME core signal closed magnetic field lines attached to the Sun at both ends. The energetic electrons may be trapped into a magnetic looplike structure and bounce back and forth between two magnetic mirrors located near the Sun. We have also examined the electron intensities and directional distributions observed by the ULYSSES/HI-SCALE experiment during the traversal and in the neighbourhood of an ICME in July 2000. The ICME was observed during the risephase of a major solar electron event. This implies a constant magnetic connection of the ICME to the Sun. Our observations indicate that energetic electrons injected at the Sun are channeled by the ICME and propagate freely along the ICME magnetic field lines to $62^{\circ} \mathrm{S}$ heliolatitude. Thus, on the basis of the presented energetic particle observations, we conclude that the most plausible interpretation of the distinct unidirectional anisotropy in the PADs (indicating electrons streaming away from the Sun are detected) is that open field lines rooted to the Sun at only one end are threading through this high-latitude ICME.

Acknowledgements. We are thankful to our HI-SCALE team colleagues for their support and encouragement.

Topical Editor R. Forsyth thanks two referees for their help in evaluating this paper.

\section{References}

Armstrong, T. P., Haggerty, D., Lanzerotti, L. J., Maclennan, C. G., Roelof, E. C., Pick, M., Simnett, G. M., Gold, R. E., Krimigis, S. M., Anderson, K. A., Lin, R. P., Sarris, E. T., Forsyth, R., and Balogh, A.: Observations by Ulysses of hot $(\sim 270 \mathrm{keV})$ coronal particles at $32^{\circ}$ south heliolatitude and 4.6 AU, Geophys. Res. Lett., 21, 1747, 1994.

Balogh, A., Beek, T. J., Forsyth, R. J., Hedgecock, P. C., Marquedant, R. J., Smith, E. J., Southwood, D. J., and Tsurutani, B. T.: The magnetic field investigation on the Ulysses mission: 
instrumentation and preliminary scientific results, Astron. Astrophys. Suppl. Ser., 92, 207, 1992.

Bame, S. J., McComas, D. J., Barraclough, B. L., Phillips, J. L., Sofaly, K. J., Chavez, J. C., Goldstein, B. E., and Sakurai, R. K.: The Ulysses solar wind plasma experiment, Astron. Astrophys. Suppl. Ser., 92, 237, 1992.

Bothmer, V., Desai, M. I., Marsden, R. G., Sanderson, T. R., Trattner, K. J., Wenzel, K.P., Gosling, J. T., Balogh, A., Forsyth, R. J., and Goldstein, B. E.: Ulysses observations of open and closed magnetic field lines within a coronal mass ejection, Astron. Astrophys., 316, 493, 1996.

Cocconi, G., Greisen, K., Morrison, P., Gold, T., and Hayakawa, S.: The cosmic ray flare effect, Nuovo Cimento, Suppl., 8, 161, 1958.

Farrugia, C. J., Burlaga, L. F., Osherovich, V. A., Richardson, I. G., Freeman, M. P., Lepping, R. P., and Lazarus, A. J.: A study of an expanding interplanetary magnetic cloud and its interaction with the Earth's magnetosphere: the interplanetary aspect, J. Geophys. Res., 98, 7621, 1993.

Gold, T.: Plasma and magnetic fields in the solar system, J. Geophys. Res., 64, 1665, 1959.

Gosling, J. T.: Coronal Mass Ejections, in 26th International Cosmic Ray Conference Invited, in: Rapporteur and Highlight Papers, vol. 516, (Eds) Dingus, B. L., Kieda, D., and Salamon, M., 59, AIP, New York, 2000.

Gosling, J. T., Hildner, E., MacQueen, R. M., Munro, R. H., Poland, A. I., and Ross, C. L.: Mass Ejections from the Sun: a view from Skylab, J. Geophys. Res., 79, 4581, 1974.

Gosling, J. T. and Roelof, E. C.: A comment on the detection of closed magnetic structures in the solar wind, Solar Phys., 39, 405, 1974

Gosling, J. T., Birn, J., and Hesse, M.: Three-dimensional magnetic reconnection and the magnetic topology of coronal mass ejection events, Geophys. Res. Lett., 22, 869, 1995.

Gosling, J. T. and Forsyth, R. J.: CME-driven solar wind disturbances at high heliographic latitudes, Space Sci. Rev., 97, 263, 2001.

Kahler, S. W. and Reames, D. V.: Probing the magnetic topologies of magnetic clouds by means of solar energetic particles, J. Geophys. Res., 96, 9419, 1991.

Klein L. W. and Burlaga, L. F.: Interplanetary magnetic clouds at 1 AU, J. Geophys. Res., 87, 613, 1982.

Kutchko, F. J., Briggs, R. P., and Armstrong, T. P.: The bidirectional particle event of 12 October 1977, possibly associated with a magnetic loop, J. Geophys. Res., 87, 1419, 1982.

Lanzerotti, L. J., Gold, R. E., Anderson, K. A., Armstrong, T. P., Lin, R. P., Krimigis, S. M., Pick, M., Roelof, E. C., Sarris, E. T., Simnett, G. M., and Frain, W. E.: Heliosphere instrument for spectra, composition and anisotropy at low energies, Astron. Astrophys. Suppl. Ser., 92, 349, 1992.

Larson, D. E., Lin, R. P., McTiernan, J. M., McFadden, J. P., Ergun, R. E., McCarthy, M., Rème, H., Sanderson, T. R., Kaiser, M., Lepping, R. P., and Mazur, J.: Tracing the topology of the 1820 October 1995, magnetic cloud with $\sim 0.1-10^{2} \mathrm{keV}$ electrons,
Geophys. Res. Lett., 24, 1911, 1997.

Lin, R. P. and Kahler, S. W.: Interplanetary magnetic field connection to the Sun during electron heat flux dropouts in the solar wind, J. Geophys. Res., 97, 8203, 1992.

Malandraki, O. E., Sarris, E. T., Kasotakis, Gr., and Sidiropoulos, N.: Study of CME structure and evolution deduced from ULYSSES/HI-SCALE energetic particle observations, Adv. Space Res., 26/5, 875, 2000a.

Malandraki, O. E., Sarris, E. T., and Trochoutsos, P.: Probing the magnetic topology of coronal mass ejections by means of Ulysses/HI-SCALE energetic particle observations, Ann. Geophysicae, 18, 129, 2000b.

Malandraki, O. E., Sarris, E. T., Lanzerotti, L. J., Maclennan, C. G., Pick, M., and Tsiropoula, G.: Tracing the magnetic topology of coronal mass ejection events by Ulysses/HI-SCALE energetic particle observations in and out of the ecliptic, Space Sci. Rev., 97, 263, 2001

Malandraki, O. E., Sarris, E. T., Lanzerotti, L. J., Trochoutsos, P., Tsiropoula, G., and Pick, M.: Solar energetic particles inside a coronal mass ejection event observed with the ACE spacecraft, J. Atmos. Sol.-Ter. Phys., 64/5-6, 517, 2002.

Marsden, R. G., Sanderson, T. R., Tranquille, C., Wenzel, K.P., and Smith, E. J.: ISEE 3 observations of low-energy proton bi-directional events and their relation to isolated interplanetary magnetic structures, J. Geophys. Res., 92, 11, 009, 1987.

Mazur, J. E., Mason, G. M., Dwyer, J. R., and von Rosenvinge, T. T.: Solar energetic particles inside magnetic clouds observed with the WIND spacecraft, Geophys. Res. Lett., 25, 2521, 1998.

Osherovich, V. and Burlaga, L. F.: Magnetic clouds, in Coronal Mass Ejections: Geophys. Monogr. Ser., vol. 99, (Eds) Crooker, N., Joselyn, J. A., Feynman, J., 157, AGU, Washington, D.C., 1997.

Palmer, I. D., Allum, F. R., and Singer, S.: Bidirectional anisotropies in solar cosmic ray events: evidence for magnetic bottles, J. Geophys. Res., 83, 75, 1978.

Pick, M., Lanzerotti, L. J., Buttighoffer, A., Sarris, E. T., Armstrong, T. P., Simnett, G. M., Roelof, E. C., and Kerdraon, A.: The propagation of sub-MeV solar electrons to heliolatitudes above $50^{\circ} \mathrm{S}$, Geophys. Res. Lett., 22, 3373, 1995.

Piddington, J. H.: Interplanetary magnetic field and its control of cosmic-ray variations, Phys. Res., 119, 589, 1958.

Rao, U. R., McCracken, K. G., and Bukata, R. P.: Cosmic ray propagation processes, 2, the energetic storm particle event, J. Geophys. Res., 72, 4325, 1967.

Richardson I. G.: Using energetic particles to probe the magnetic topology of ejecta, in: Coronal Mass Ejections, Geophys. Monogr. Ser., vol. 99, (Eds) Crooker, N., Joselyn, J. A., Feynman, J., 189, AGU, Washington, 1997.

Sarris, E. T. and Krimigis, S. M.: Evidence for solar magnetic loops beyond 1 AU, Geophys. Res. Lett., 9, 167, 1982.

Simnett, G. M.: Energetic particle characteristics in the highlatitude heliosphere near solar maximum, Space Sci. Rev., 97 231, 2001. 